\title{
Improving the Accuracy of Ontology Alignment through Ensemble Fuzzy Clustering
}

\author{
Nafisa Afrin Chowdhury and Dejing Dou \\ Department of Computer and Information Science, \\ University of Oregon, Eugene, OR 97403, USA \\ \{nafisa, dou\}@cs. uoregon.edu
}

\begin{abstract}
Automatic ontology alignment tools perform matching between the concepts of two ontologies and provide a similarity measure for each pair of aligned concepts. However, none of the existing tools are perfect and multiple alignment tools produce varying similarity measures for a certain alignment. Also, the similarity measures provided by an alignment may not be helpful enough for indicating the degree of reliability. While using a random alignment tool we noticed that some quality alignments are given medium or even low similarity measures, and that causes the user ignoring those alignments. In this study we have proposed an ensemble model of ontology alignment that aggregates multiple alignment tools with the help of Fuzzy C Means clustering and Type 2 Fuzzy Membership Functions. We have shown that our approach helps the user to choose the best alignment results which has not been obtained by any other alignment tools we experimented with.
\end{abstract}

Keywords: ontology alignment, uncertainty, Fuzzy C Means clustering (FCM), Interval Type 2 Fuzzy Membership Function, Ensemble Fuzzy Clustering.

\section{Introduction}

Automatic ontology alignment tools reduce the tedious manual job of establishing the correspondences between two ontologies [1. Most of the available automatic tools provide a similarity metric (generally $0.0-1.0$ ) associated with the alignment based on the algorithm being used. The problem arises when a user is looking for quality alignments in order to use in his application. It is expected that an alignment with low similarity measure is not as helpful as an alignment with higher value. However, there is no absolute threshold value that can distinguish quality and poor alignments. We observed that the automatic alignment tool Falcon $\mathrm{AO} 2$ that uses an integrated alignment algorithm of linguistic and graphical matcher, suffers this problem. When we applied two ontologies of camera domain we found only nine alignments with similarity measure more than 0.7 , as there was a default threshold value 0.7 . However, when we changed the threshold value to 0.1 in the source code, we found several other quality alignments even though they had lower similarity measure. Having 
the discrete threshold of 0.7 obscured some of the alignments such as "lens""lenGroup", "PurchaseableItem"- "PurchasebleItem" etc. In our ensemble ontology alignment model we considered threshold value zero in order to obtain all possible alignments from an alignment tool.

Another problem is, the alignment tools using different similarity algorithms almost never agree to each other and this causes ambiguity of selecting the "appropriate" tool. We observed running a pair of bibliographic ontologies in three ontology alignment tools: Falcon AO [2], Anchor Flood [4] and OLA [5]. We found most of the alignments received three different similarity measure values from them. For example, an alignment "hasInstitution"-_institution" received $0.97,1.0$ and 0.33 from those tools respectively. Apparently this alignment is a strong one according to first two of the tools but weak based on the judgment of third the tool. Now, the question arises which similarity value is correct?

In order to address the issue of deciding the quality of an alignment our Ensemble Fuzzy Clustering approach incorporated fuzzy membership functions. Instead of specifying a discrete boundary of "quality" and "poor" alignments, fuzzy membership functions specifies the possibility of an alignment to be a member of certain class [3. In most cases fuzzy membership functions are established by human experts. However, as it is expensive to have human experts we built membership functions automatically with the help of Fuzzy C Means (FCM) clustering [7. The reason of choosing FCM is that it provides us with overlapping class boundaries, which is more likely a fuzzy membership function. When we obtained membership functions automatically from multiple alignment tools, we aggregated the results using Interval Type 2 Fuzzy Membership Functions (IT2MF) 3. IT2MF is described as a blurred type 1 fuzzy membership function [3], where the degree of membership function will be represented as a range of available membership grades.

The rest of this paper is organized as follows: we will study several other research publications that expected to illuminate in related areas(section 2). In section 3 we illustrated our own ensemble model of choosing the best alignments in details. The section 4 demonstrates our experiments in two cases(bibliographic text and family). In the last section we discussed our contributions in this paper and future works.

\section{Related Works}

In order to address the problem of alignment tool selection Eckert et al [6] developed a framework using machine learning technique. This work was similar to ours in the sense that it also tried to aggregate the results of the alignment tools instead of using a single. The major difference with our approach is that, in this work the authors used discrete values to evaluate an alignment and we used floating point similarity measure values. Also, unlike our clustering the authors of this paper assigned human specified class labels along with the alignments. However, this causes the same problem of discrete threshold values as we described in the introduction. 
There are not many works had been accomplished that addressed uncertainty in ontology alignment using fuzzy logic. Among the very few Niwattanakul et al proposed an approach to classify an ontology similarity based on fuzzy membership function [8]. Here the authors divided the alignments into some classes between most similar to least similar. In our approach we avoided using classification technique as our alignments did not have class labels.

Another work with slightly different objective proposed by Tordai et al studied longer chains of mappings when more than two ontologies are aligned 10]. This paper also tried to divide the alignments into several groups based on their similarity. The authors used sampling methods for evaluating the alignments and assign them into matching groups by human experts. Our approach outperforms in this regard as there is no human expert intervention required. We used a Fuzzy based clustering method that generates the grouping in membership function automatically.

\section{Methodology}

In our Ensemble Fuzzy Clustering Approach, we hypothesized a fuzzy membership function (MF) that will address the degree of uncertainty for all resultant alignments. Here an alignment will have a membership value for each of the classes and all the membership values of a particular alignment will be added up to one, which indicates the valid existence of the alignment. Figure 1 portrays our model of Ensemble Fuzzy Clustering.

In order to build MFs automatically clustering was more promising than classification methods, as there was no class labels associated with the alignments. Also, MFs require fuzzy or overlapping class boundaries which is unavailable in generic clustering methods (like K-means). We found Fuzzy C Means (FCM) clustering [7] 11] provides overlapping class boundaries where a data instance can belong to more than one clusters. We applied FCM on the similarity measure values provided by the alignment tools and, obtained the membership values for each of the alignments. We also obtained the center values of those clusters. Figure 1 shows how the membership functions are being generated from $\mathrm{n}$ alignment tools. If there are three clusters, we can easily distinguish the cluster with highest center value as the cluster of most quality alignments.

In figure 1 there are $\mathrm{p}$ membership functions generated from each of the tools. For a particular cluster we can combine the n MFs generated from $n$ tools by applying the Interval Type 2 Fuzzy Logic theory. In our approach, for a particular cluster we constructed an IT2MF, where the membership degree interval of a single alignment was the interval of its observed maximum and minimum membership values. We will see examples of constructing IT2MF in the case studies section.

Now the question is how we can infer the best alignments from the IT2MF we developed in Fig 1. An alignment having longer interval denotes that the alignment tools did not agree upon its strength. So the alignment that has longer interval is more uncertain than the alignment with shorter interval. This intuition 


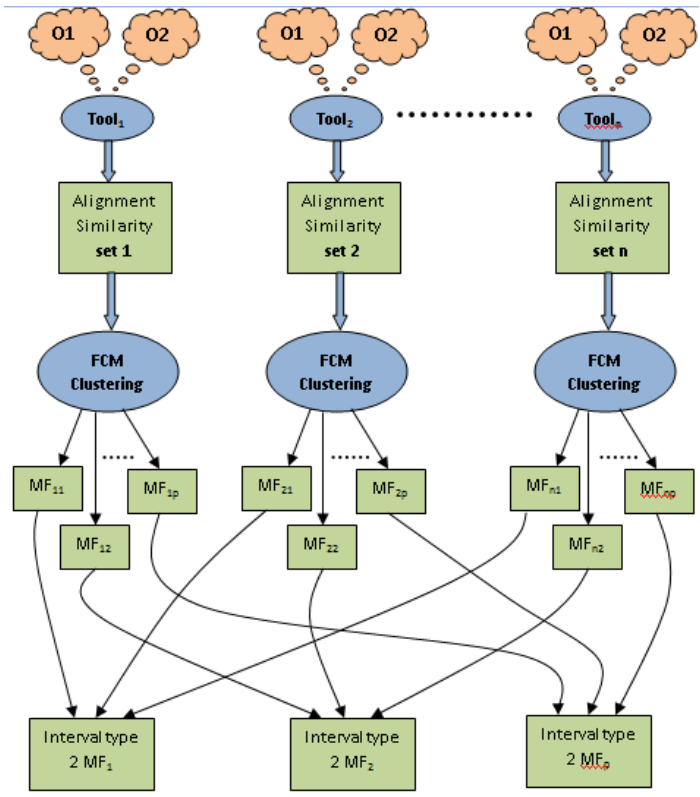

Fig. 1. Generating membership functions automatically by applying FCM on the results of the alignment tools. Here $\mathrm{n}$ is the number of alignment tools, $\mathrm{p}$ is the number of clusters or the membership functions. $O_{1}$ and $O_{2}$ are two ontologies being aligned.

gives us a way of comparing the alignments based on their uncertainties. Again, the alignment that has higher interval mean is stronger as it denotes that most of the alignment tools suggested this one higher similarity measure. So this alignment is most likely to be a strong one. At this point we needed to develop an equation that balances the two concepts of uncertainty(interval width) and strength(interval mean). We introduced "potential" as a quality metric of an alignment. The equation 1 is a weighted harmonic mean formula that provides more emphasis on the interval mean than the interval (width). The reason of using weighted harmonic mean instead of generic one is that, intuitively it is more significant to have higher interval mean rather than having lower uncertainty. If an alignment shows higher interval mean, it is more likely to be in the strong cluster.

$$
P_{i}=\left(1+\beta^{2}\right) \frac{\text { mean }_{i} * \text { interval }_{i}}{\text { mean }_{i}+\beta^{2} \text { interval }_{i}}
$$

Where, $P_{i}$ is the potential of the alignment with index i. $\beta$ is a constant. Interval $_{i}$ is the interval of the ith alignment. Mean $i$ is the arithmetic mean of Interval $_{i}$. The potential value reflects both the uncertainty and the strength of an alignment. Therefore, the best alignment has the highest mean and least interval(width). 


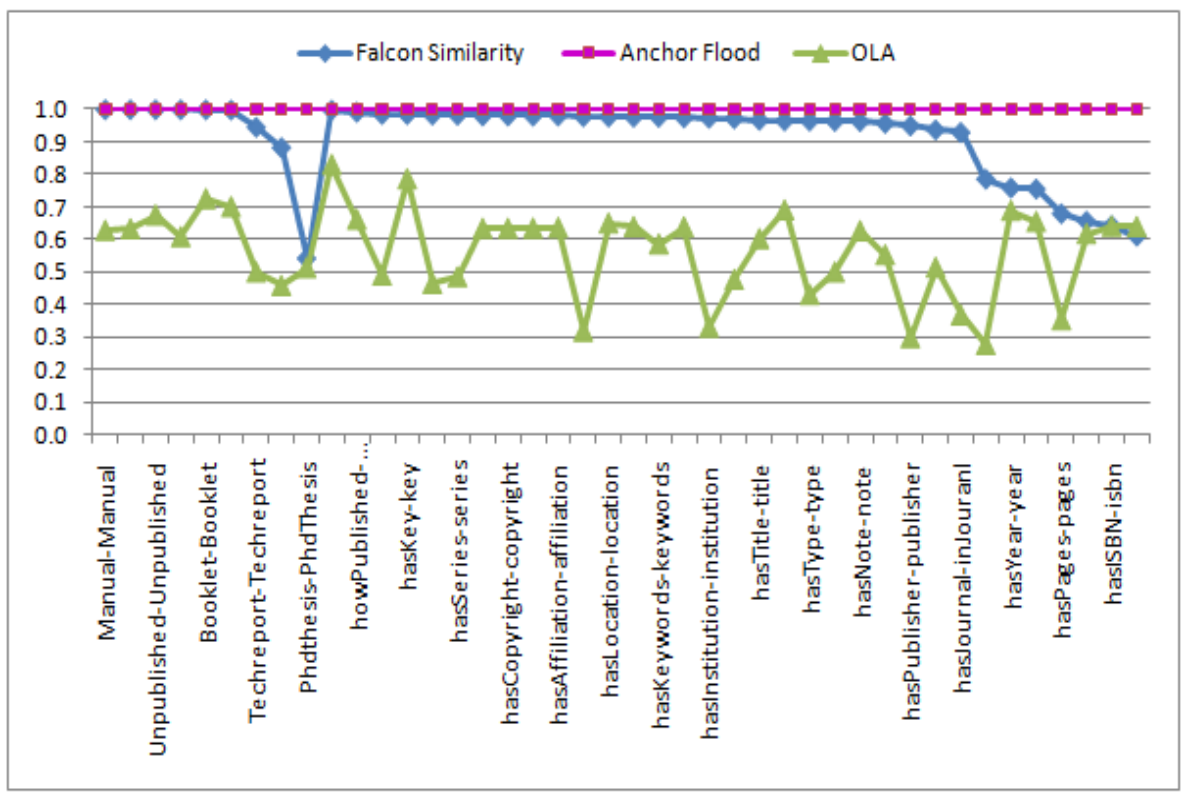

Fig. 2. Alignments with their similarity measure values found after running three ontology alignment tools for a pair of bibliographic text ontologies

\section{Case Studies}

\subsection{Bibliographic Text Ontologies}

We have experimented with a pair of ontologies in bibliographic text and three automatic alignment tools Falcon AO 2], Anchor Flood [4] and OLA [5. Figure 2 shows the alignment results found after feeding the two OWL files of bibliographic text to all three of the alignment tools. For simplicity we restricted ourselves considering only the 42 alignments found common in all three of the tools. According to our methodologies we applied FCM. Fig. [3]shows the degree of membership values found after applying FCM [11] on the similarity measure values of Falcon AO [2]. In this figure, if we draw a line connecting all the membership values of strong cluster, we will obtain the membership function for this cluster. Similar membership functions can be drawn for medium and weak clusters.

We performed similar operations on the alignment similarity measure values obtained from other tools. As the tools did not agree upon the similarity measure values of the alignments, for a particular cluster we obtained very different MFs from them. In order to aggregate multiple alignment tools we applied IT2MF on these MFs. We found alignment number 11 ("howPublished"- "howPublished") and alignment number 13 ("hasKey"-"key") have membership degree interval from 0.2 to 1.0 and 0.93 to 1.0 respectively. According to our methodology, number 13 is stronger and less uncertain than number 11, as it has higher mean and shorter interval width. For strong cluster figure 4 clearly shows how the 


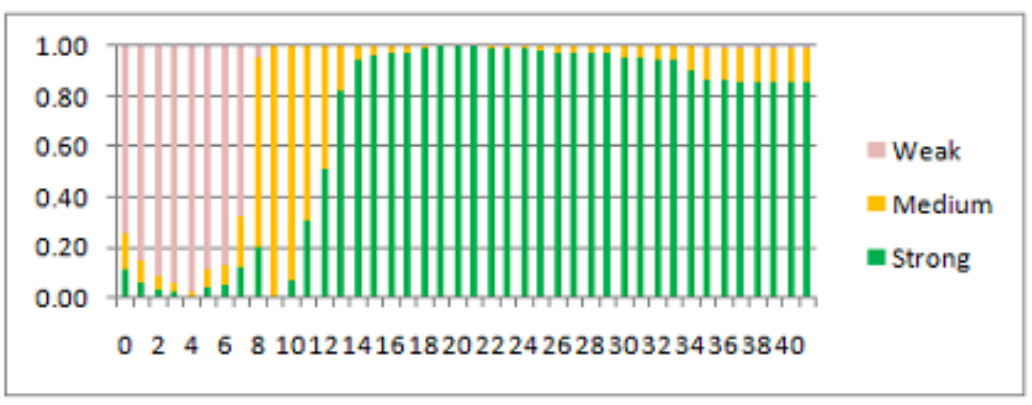

Fig. 3. Membership functions for three clusters: weak, medium and strong generated after applying FCM on Falcon similarities

uncertainty range looks like for the 42 alignments we considered. We excluded Anchor Flood [4], as it provided same membership degree for all the alignments and this will moderate the actual potential value of an alignment.

In order to sort our alignments based on their strength and uncertainty we calculated their potential values according to the equation 1. Here we have chosen $\beta=10.0$, as it distributes the potential value in a way that the interval mean gets much higher emphasis than the interval (width). We found that alignments with ambiguous similarity measure (very high in one tool and very low in other) achieved a moderate potential value that respects all the algorithmic views of those tools we considered.

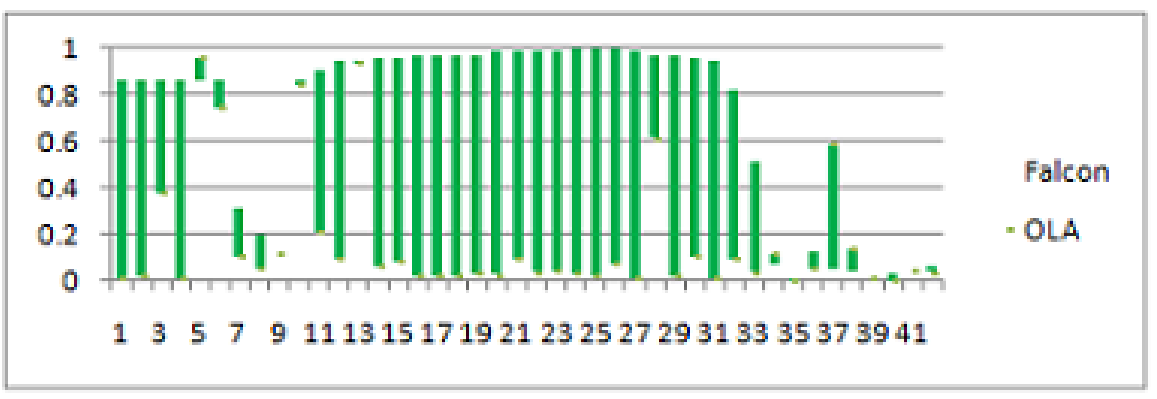

Fig. 4. Interval type 2 membership function for strong cluster considering two alignment tools. As Anchor flood assigned 1.0 for all of the alignments we exclude this tool here to clarify the necessity of considering mean in the selection criteria.

\subsection{Family Ontologies}

We performed another case study with a pair of ontologies in Family domain. We obtained 14 alignments and their similarity values after running the three alignment tools with these ontologies. We repeated our methodologies in the same way as we performed in the case of bibliographic text. Unfortunately, these two ontologies were similar enough to build a sparse membership function like 
bibliographic text. Most of the alignments received high range of uncertainty (more than 0.6), because the similarity measures of the alignments were high (more than 0.98) and we tried to divide them into three clusters in between 0.98 and 1.0. Therefore the centers of those clusters were close enough and this made the clustering decision difficult.

We could not find any set of alignments that could be used as a golden standard for this pair of ontologies. As they were small enough we performed the alignment ourselves and compared after sorting the alignments based on their calculated potential values. We found no significant difference between our approach and other alignment tools for the case of family ontologies.

\subsection{Results}

In order to justify our theory of calculating potentials we compared our results with the EON [12] ontology alignment contest results as our golden standard. But the problem was all existing alignments in EON have similarity value 1.0, whereas we have floating point similarity and potential values. For doing the comparison we termed an alignment "wrong" if it does not exist in the EON result. We sorted the 42 alignments (figure 2) based on their calculated potential values and counted the number of "wrong" alignments. Table 4.3 summarizes the results of comparing our method with other existing alignment tools. It clearly shows that sorting the alignments based on their potential values provides better results than all other alignment tools we considered, because the potential value has been calculated by aggregating multiple alignment tools. We have not included results of sorting the alignments based on Anchor Flood, as it assigns same similarity measure(1.0) for all.

Table 1. Comparing the number of wrong alignments found after sorting the alignments in descending order based on different methods. The table reports the number of wrong alignments present in the first 25 .

\begin{tabular}{lll}
\hline Sorted based on & \multicolumn{2}{l}{ Wrong Position } \\
\hline Potential & 1 & 15th \\
Falcon similarity & 2 & 2nd and 19th \\
OLA similarity & 2 & 12th and 19th \\
Average of 3 tools 3 & $9 t h, 13 t h$ and $21 s t$ \\
\hline
\end{tabular}

\section{Conclusion}

Most of the alignment tools provide a similarity measure but it is hard to justify the quality of an alignment only by looking at its similarity measure. Also this similarity measure value is different in different alignment tools, that raises ambiguity and uncertainty. This paper provides an ensemble model that aggregates the results of multiple ontology alignment tools. As described in the methodologies section we applied Fuzzy C Means clustering and IT2MF in order to obtain 
an aggregated fuzzy membership function that combines the results of all alignment tools we considered. Finally we introduced a formula for calculating the potential of an alignment, where the potential addresses both the strength and uncertainty of an alignment. We have also proved that if we sort the alignments based on their potential it performs better than all other alignment tools. We would like to extend this work by applying more ontology alignment tools in the future.

\section{References}

1. Noy, N.F., Musen, M.A.: PROMPT: Algorithm and Tool for Automated Ontology Merging and Alignment. In: Proceedings of the National Conference on Artificial Intelligence, pp. 450-455 (2000)

2. Jian, N., Hu, W., Cheng, G., Qu, Y.: Falcon-ao: Aligning ontologies with falcon. In: K-Cap 2005 Workshop on Integrating Ontologies, pp. 87-93 (2005)

3. Mendel, J.M.: Uncertainty Rule-Based Fuzzy Logic Systems. Prentice Hall PTR, Upper Saddle River (2001)

4. Seddiqui, M.H., Aono, M.: An effcient and scalable algorithm for segmented alignment of ontologies of arbitrary size. Web Semant. 7(4), 344-356 (2009)

5. Euzenat, J., Valtchev, P.: Similarity-Based Ontology Alignment in OWL-Lite. In: ECAI 2004, pp. 333-337 (2004)

6. Eckert, K., Meilicke, C., Stuckenschmidt, H.: Improving Ontology Matching Using Meta-Level Learning. In: Aroyo, L., Traverso, P., Ciravegna, F., Cimiano, P., Heath, T., Hyvönen, E., Mizoguchi, R., Oren, E., Sabou, M., Simperl, E. (eds.) ESWC 2009. LNCS, vol. 5554, pp. 158-172. Springer, Heidelberg (2009)

7. Dunn, J.C.: A Fuzzy Relative of the ISODATA Process and Its Use in Detecting Compact Well-Separated Cluster. Cybernetics and Systems 3(3), 32-57 (1973)

8. Niwattanakul, S., Martin, P., Eboueya, M., Khaimook, K.: Ontology Mapping based on Similarity Measure and Fuzzy Logic. In: Proceedings of World Conference on E-Learning in Corporate, Government, Healthcare, and Higher Education, Quebec City, Canada, 2007, pp. 6383-6387 (2009)

9. Ding, Z., Peng, Y., Pan, R.: A Bayesian Approach to Uncertainty Modelling in OWL Ontology. In: Proceedings of the International Conference on Advances in Intelligent Systems - Theory and Applications, Luxembourg, Germany (2004)

10. Tordai, A., Ghazvinian, A., van Ossenbruggen, J., Musen, M.A., Noy, N.F.: Lost in Translation? Empirical Analysis of Mapping Compositions for Large Ontologies. In: The Fifth International Workshop on Ontology Matching (OM 2010), at ISWC 2010, Shanghai, China (2010)

11. Hathaway, R.J., Davenport, J.W., Bezdek, J.C.: Relational duals of the c-means algorithms. Pattern Recognition 22, 205-212 (1989)

12. EON Ontology Alignment Contest, http://oaei.ontologymatching.org/2004/Contest/ 